AN. MED. INTERNA (Madrid) Vol. 18, N. $^{\circ} 9$, pp. $459-463,2001$

\title{
Valoración de isoamilasas e isolipasas en el suero de pacientes con insuficiencia renal
}

\author{
M. R. SÁNCHEZ NAVARRO, E. FERNÁNDEZ-CONDE, C. SAMANIEGO \\ Departamento de Bioquímica Clínica. Hospital Universitario San Cecilio. Granada
}

\author{
ISOAMYLASES AND ISOLIPASES VALORATION IN THE SERUM \\ FROM PATIENTS WITH RENAL INSUFFICIENCY
}

\begin{abstract}
RESUMEN
Objetivo: Estudiar la utilidad clínica de la determinación de isoamilasas e isolipasas en el suero de pacientes con insuficiencia renal.

Material y métodos: Determinamos los niveles séricos de isoamilasas e isolipasas en un grupo de 68 pacientes con enfermedad renal, 32 con insuficiencia aguda y 36 con fallo renal crónico y sometidos a hemodiálisis, comparándose los resultados con los de una población de 44 adultos sanos. Utilizamos un nuevo método para la determinación sérica de isolipasas basado en su separación electroforética sobre gel de agarosa. Dos formas de lipasa, L1 y L2, fueron identificadas por este método y cuantificadas por densitometría.

Resultados: Encontramos un aumento significativo de la isoamilasa pancreática P2 y del cociente isoenzimatico P/S tanto en los pacientes agudos $(p<0,001)$ como en los crónicos $(p<0,05)$. En ambos grupos la actividad de la isolipasa L1 y el cociente L1/L2 se mostraron significativamente elevados $(\mathrm{p}<0,01)$. Estudiamos la relación entre isoamilasas e isolipasas estableciendo el cociente $\mathrm{P} 2 / \mathrm{L} 2$ (rango normal $<0,6$ ) encontrando, en los dos grupos patológicos, valores significativamente elevados respecto al grupo control $(\mathrm{p}<0,001)$ y una correlación positiva y estadísticamente significativa entre los cocientes P2/L2 y P/S ( $\mathrm{r}=0,76$, $\mathrm{p}<0,05$ en pacientes agudos; $\mathrm{r}=0,58, \mathrm{p}<0,05$ en crónicos).

Conclusión: El estudio combinado de isoamilasas e isolipasas séricas podría ser un marcador efectivo para el diagnóstico y evolución de la pancreatitis asociada al fallo renal agudo o crónico.
\end{abstract}

PALABRAS CLAVE: Isoamilasas. Isolipasas. Insuficiencia renal.

\begin{abstract}
Objective: The aim of this study is to test the utility os serum isoamy lases and isolipases as determinated from patients with renal insuffi ciency.

Material and methods: Serum levels of isoamylases and isolipases were determined in a group of sixty-eight patients with renal disease, 32 of them suffering acute insufficiency and 36 with chronic renal failure undergoing regular hemodialysis, results obtained were compared from a population of 44 healthy adults. We used a new method for isolipases determination in serum based on its separation on agarose gel. Two forms of lipase, L1 and L2, were identified by this method and quantita ted by densitometry.

Results: Were found a significant increase of pancreatic isoamylase $P 2$ and $P / S$ isoenzymatic ratio in acute patients $(p<0.001)$ as chronic ( $p<0.05)$. In both groups, the isolipase L1 activity and L1/L2 isoform ratio were showed significantly elevated $(p<0.01)$. We studied the rela tionship between isoamylases and isolipases establishing the P2/L2 ratio (normal range <0.6) showing, in the two pathologic groups, significantly elevated values compared with the control group $(p<0.001)$ and a positi $v e$ and significant correlation between the P2/L2 and $P / S$ isoform ratios ( $r=0.76, p<0.05$ in acute pantients; $r=0.58, p<0.05$ in chrónic patients).

Conclusion: The combined study of serum levels of isoamylases and isolipases could be an effective marker for diagnosis and evolution of associated pancreatitis with acute or chronic renal failure.
\end{abstract}

KEY WORDS: Isoamylases. Isolipases. Renal insufficiency.

Sánchez Navarro MR, Fernández-Conde E, Samaniego C. Valoración de isoamilasas e isolipasas en el suero de pacientes con insuficiencia renal. An Med Interna (Madrid) 2001; 18: 459-463.

\section{INTRODUCCIÓN}

La enfermedad pancreática es una conocida complicación del fallo renal; signos evidentes de pancreatitis se han encontrado en el $2 \%$ de los pacientes urémicos aunque en muchas ocasiones el daño pancreático es subclínico (1-4). En 1948, Baggestos llamó la atención sobre el hecho de que en el 50\% de los pacientes con insuficiencia renal crónica (IRC) se podían demostrar anormalidades histológicas en el páncreas: dila- tación ductal, fibrosis periductal e intersticial, destrucción de células acinares, etc. (5-7).

En la práctica clínica, las concentraciones séricas de las enzimas pancreáticas, amilasa (EC 3.2.1.1) y lipasa (EC 3.1.1.3), se usan de forma generalizada como pruebas de detección selectiva de pancreatitis en el paciente con dolor abdominal agudo. Elevaciones séricas de los niveles de amilasa y lipasa se han observado en el 60-80\% de los enfermos urémicos; este incremento es generalmente moderado cuando

Trabajo aceptado: 19 de marzo de 2001

Correspondencia: Ma Rosa Sánchez Navarro. Departamento de Bioquímica. Facultad de Medicina. Avda. de Madrid. 18012 Granada. 
el fallo renal no coexiste con una inflamación pancreática y se ha relacionado con el grado de deterioro de la función renal, dado que, ambas enzimas son filtradas por el glomérulo el fallo renal condicionaría el aumento de sus niveles séricos. Actualmente, el significado de la elevación sérica de éstas y otras enzimas pancreáticas en el enfermo renal, no esta completamente claro (8-14).

La lipasa pancreática está presente en el suero bajo dos formas conocidas como L1 y L2, fácilmente identificables por electroforesis en virtud a su diferente carga superficial (1517). Así como, la determinación de isoenzimas de amilasa ha sido de gran utilidad diagnóstica al permitir descartar hiperamilasemias de causa no pancreática, el estudio de las diferentes formas de lipasa podría proporcionar información diagnóstica más específica que la simple medición de la actividad enzimática total (18-20).

El objetivo del presente trabajo ha sido valorar la utilidad clínica del estudio combinado de isolipasas e isoamilasas de tipo pancreático tanto en el diagnóstico como en la frecuencia y severidad de la pancreatitis asociada al fallo renal agudo y crónico.

\section{MATERIAL Y MÉTODOS}

\section{PACIENTES}

Isoamilasas e isolipasas se estudiaron en un total de 112 individuos clasificados en dos grupos: un grupo, formado por 44 adultos (24 mujeres y 20 varones; media de edad 41 años; rango 18-65) aparentemente sanos, normoamilasémicos y normolipasémicos, que sirvieron para establecer valores de referencia y se utilizaron como control, y un grupo patológico formado por 68 pacientes, que fueron seleccionados en base a parámetros clínicos, bioquímicos y valoración objetiva de la enfermedad mediante pruebas complementarias adecuadas, compatibles con una insuficiencia renal; 32 pacientes (15 mujeres y 17 hombres; media de edad 46 años; rango 17- 72) con signos y síntomas de Insuficiencia renal aguda (IRA) y 36 pacientes (16 mujeres y 20 hombres; media de edad 48 años; rango 26-74) con Insuficiencia renal crónica (IRC) y sometidos a hemodiálisis. Todos los pacientes mostraron una elevación moderada de las actividades séricas de amilasa y lipasa pero ninguno presentó signos clínicos de pancreatitis aguda.

\section{MÉTODOS ANALÍTICOS}

Parámetros bioquímicos. Urea, creatinina, actividad total de amilasa y lipasa se midieron a $37{ }^{\circ} \mathrm{C}$, en Autoanalizador Hitachi 917 con reactivos Boehringer Mannheim (Automated Analysis for BM/Hitachi 917; Mannheim, F.R.G.).

Isoenzimas de amilasa. La separación de isoamilasas se realizó con el método Paragon/Beckman, utilizando kits Isoamyl (Analis, Namur, Belgium). Se utilizó gel de agarosa tamponado en buffer (Tris/Borato, $\mathrm{pH}$ 6,95). La electroforesis tuvo lugar a 150 voltios durante 30 minutos; una vez terminada, se incubaron los geles con un substrato específico para la enzima. La cantidad relativa de las bandas se cuantificó a 600 nanometros en un densitómetro Appraise de Beckman (Beck man Instruments, Inc.) y la cantidad de cada isoenzima se calculó como porcentaje de la actividad enzimática total.
Isoenzimas de Lipasa. Se utilizó el sistema Paragon Electrophoresis System de Beckman (Beckman Instruments, Fullerton, $C A$ ) y se adaptaron los geles de agarosa para isoenzimas de creatin kinasa (CK) P/N 655930 para la separación de las isoformas de lipasa. Como substrato, se utilizaron los reactivos Lipase-PS (Sigma Diagnostics Co., St Louis) destinados a la determinación cuantitativa, cinética, de la actividad de la lipasa pancreática en suero. La electroforesis tuvo lugar a 100 voltios y se mantuvo durante 25 minutos. Para la visualización de las bandas se incubaron los geles con substratocolor de lipasa en caja de incubación oscura y en cámara húmeda a $45^{\circ} \mathrm{C}$ durante 30 minutos. Después de la incubación los geles fueron evaluados visualmente y en un densitómetro Appraise a 540 nanometros.

\section{MUESTRAS}

Jugo pancreático. Procedente del drenaje de una fístula pancreática, con una actividad de amilasa de $100.000 \mathrm{U} / \mathrm{L}$ aprox., un patrón electroforético conocido y formado, exclusivamente, por isoamilasas pancreáticas $(\mathrm{P} 1=12 \%, \mathrm{P} 2=68 \%$ y $\mathrm{P} 3=20 \%$ ) y una actividad total de lipasa de $60.000 \mathrm{U} / \mathrm{L}$ aprox.; homogeneizado, centrifugado, diluído con un suero normal inactivado previamente al calor durante 1 hora a $56^{\circ} \mathrm{C}$ y conservado en alícuotas a $-20^{\circ} \mathrm{C}$.

Saliva. Saliva fresca humana, con un contenido de amilasa de $16.000 \mathrm{U} / \mathrm{L}$ aprox. Y un patrón electroforético formado por isoamilasas salivales $(\mathrm{S} 2=49 \%, \mathrm{~S} 3=34 \%, \mathrm{~S} 4=17 \%$ ) centrifugada, diluída con agua destilada y conservada en alícuotas a $20{ }^{\circ} \mathrm{C}$. Las muestras de jugo pancreático y de saliva fueron utilizadas como controles en la separación electroforética de isoenzimas.

Muestras de sangre. Se utilizaron muestras de suero que se obtuvieron siguiendo el proceso habitual del laboratorio. Las muestras con evidencia de hemólisis fueron rechazadas. Cuando fue posible se procesaron inmediatamente, cuando no, se mantuvieron entre 2 y $4{ }^{\circ} \mathrm{C}$ durante un período máximo de tres días. Una alícuota de cada muestra fue inmediatamente congelada a $-20{ }^{\circ} \mathrm{C}$ para la determinación de isoenzimas de lipasa con el objeto de evitar la interferencia en los resultados de una posible transformación in vitro de una forma de lipasa a otra. Las descongelaciones repetidas fueron evitadas rigurosamente. En los pacientes crónicos la extracción de la muestra tuvo lugar antes de la diálisis

Valores de referencia. Los valores de referencia se calcularon a partir del valor medio $\pm 2 \mathrm{DE}$ de los valores de isolipasas encontrados en una población de 44 individuos (24 mujeres y 20 varones) adultos, aparentemente sanos.

Análisis estadístico. Las variables cuantitativas se expresan en función de su media y desviación estándar $(\bar{X} \pm D E)$. Se utiliza el test paramétrico de la " $t$ " de Student para comparación de muestras independientes y se aplicó el método de regresión lineal " $r$ " de Pearson para estudiar la correlación entre variables, estableciéndose el nivel de significación en un valor de $p<0,05$. Los datos se procesaron con el paquete estadístico Rsigma para PC (21). La bibliografía se obtuvo gracias al Servidor de la Hemeroteca de la Facultad de Medicina de Granada, utilizando el método on-line y recuperando las publicaciones sobre el tema incluídas en el sistema internacional MEDLINE desde los años 1971 a 1999. 


\section{RESULTADOS}

En el grupo control la actividad total de amilasa plasmática fue de $117 \pm 26 \mathrm{U} / \mathrm{L}$, la actividad de lipasa pancreática $77 \pm$ $43 \mathrm{U} / \mathrm{L}$, y los niveles de urea y creatinina inferiores a $50 \mathrm{mg} / \mathrm{dl}$ y $1 \mathrm{mg} / \mathrm{dl}$ respectivamente.

Los pacientes con insuficiencia renal aguda presentaron cifras elevadas de urea $(194 \pm 49 \mathrm{mg} / \mathrm{dl})$ y creatinina $(6 \pm 2,9$ $\mathrm{mg} / \mathrm{dl}$ ); actividades séricas de amilasa (326 $\pm 156 \mathrm{U} / \mathrm{L})$ significativamente elevadas con respecto al grupo control $(p<0,001)$ y perfiles isoenzimáticos característicos: isoamilasa pancreática P2 muy aumentada, isoenzimas salivales S2 y S3 disminuidas y relación $\mathrm{P} / \mathrm{S}$ significativamente aumentada con relación al grupo control $(\mathrm{p}<0,001)$.

Los pacientes con Insuficiencia renal crónica, mostraron cifras de urea $(125,5 \pm 38 \mathrm{mg} / \mathrm{dl})$, creatinina $(8,7 \pm 2,7 \mathrm{mg} / \mathrm{dl})$ y una actividad total de amilasa $(278 \pm 112 \mathrm{U} / \mathrm{L})$, aumentada con respecto al grupo control $(\mathrm{p}<0,001)$. En cuanto a los perfiles isoenzimáticos, los porcentajes de isoamilasas de tipo-pancreático disminuyeron y las isoamilasas tipo-salival aumentaron significativamente en relación con los pacientes agudos $(\mathrm{p}<0,001)$, pero tanto las isoamilasas $\mathrm{P} 2$, como el cociente $\mathrm{P} / \mathrm{S}$ se mantuvieron significativamente elevados en comparación con el grupo control $(\mathrm{p}<0,05)$. En la tabla I se reflejan los valores porcentuales medios y la desviación estándar (DE) de las isoenzimas de amilasas y el cociente P/S, encontrados en todos los grupos estudiados.

TABLA I

\begin{tabular}{cccccc}
\multicolumn{6}{c}{ ACTIVIDAD SÉRICA DE ISO AM ILASAS EN LO S GRUPO S } \\
ESTUDIADOS. VALO RES M EDIO S \pm DE \\
\hline \multicolumn{6}{c}{ Isoamilasas } \\
\hline & $n$ & $\%$ P2 & $\%$ S2 & $\%$ S3 & Cociente P/S \\
\hline Control & 44 & $37,7 \pm 8,1$ & $57,6 \pm 8,5$ & $4,7 \pm 2,8$ & $0,62 \pm 0,19$ \\
RA & 32 & $57,6 \pm 11^{* *}$ & $37,9 \pm 9,2^{* *}$ & $4,5 \pm 3,6$ & $1,64 \pm 0,99 * *$ \\
RC & 36 & $42,6 \pm 12^{*}$ & $48,6 \pm 9,4^{*}$ & $8,8 \pm 6,1$ & $0,81 \pm 0,44^{*}$ \\
\hline
\end{tabular}

a Expresadas como porcentaje de la actividad total de amilasa.

* $\mathrm{p}<0,05$; ** $\mathrm{p}<0,001$.

Tanto en los pacientes con IRA como los pacientes con IRC y hemodializados, la actividad total de lipasa pancreática mostró valores significativamente elevados, $303 \pm 162 \mathrm{U} / \mathrm{L}$ y $222,7 \pm 152 \mathrm{U} / \mathrm{L}$ respectivamente, $(\mathrm{p}<0,001)$. Los perfiles isoenzimaticos de lipasa se caracterizaron, en ambos grupos, por una fracción L1 significativamente aumentada, una fracción L2 disminuida y un cociente L1/L2 significativamente aumentado respecto a los individuos sanos $(\mathrm{p}<0,01)$. La tabla II muestra los valores porcentuales de isolipasas y los cocientes L1/L2 y P2/L2.

Para estudiar la relación existente entre los valores de isoamilasas e isolipasas encontrados en los dos grupos de enfermos renales, se estableció el cociente P2/L2 que relaciona, en cada paciente, la fracción pancreática $\mathrm{P} 2$ de la amilasa con la fracción L2 de la lipasa. Los valores de referencia para este cociente se calcularon a partir del valor medio $\pm 2 \mathrm{DE}$, sobre
TABLA ॥

ACTIVIDAD SÉRICA DE ISO LIPASAS EN LOS GRUPOS ESTUDIADOS. VALORES MEDIOS \pm DE

\begin{tabular}{|c|c|c|c|c|c|}
\hline & \multicolumn{3}{|c|}{ Isolipasasa } & \multirow{2}{*}{$\begin{array}{c}\text { Cociente } \\
\text { L1/L2 }\end{array}$} & \multirow{2}{*}{$\begin{array}{c}\text { Cociente } \\
\text { P2/L2 }\end{array}$} \\
\hline & $n$ & $\%$ L1 & $\%$ L2 & & \\
\hline $\begin{array}{l}\text { Control } \\
\text { IRA } \\
\text { IRC }\end{array}$ & $\begin{array}{l}44 \\
32 \\
36\end{array}$ & $\begin{array}{c}8,8 \pm 5,6 \\
14,3 \pm 10^{*} \\
14,6 \pm 9,3 *\end{array}$ & $\begin{array}{c}91,2 \pm 5,6 \\
85,7 \pm 10^{*} \\
85,4 \pm 9,3^{*}\end{array}$ & $\begin{array}{l}0,10 \pm 0,07 \\
0,18 \pm 0,14^{*} \\
0,19 \pm 0,14^{*}\end{array}$ & $\begin{array}{c}0,41 \pm 0,09 \\
0,67 \pm 0,13^{* *} \\
0,53 \pm 0,16^{* *}\end{array}$ \\
\hline
\end{tabular}

a Expresadas como porcentaje de la actividad total de lipasa.

* $\mathrm{p}<0,01 ; * * \mathrm{p}<0,001$.

los encontrados en el grupo control, estableciéndose el rango normal en un valor $\mathrm{P} 2 / \mathrm{L} 2<0,6$. Ambos grupos patológicos mostraron valores significativamente elevados de P2/L2 respecto al grupo control $(\mathrm{p}<0,001)$.

Se estimó el coeficiente de correlación entre variables encontrándose una correlación positiva y estadísticamente significativa entre los cocientes P/S y P2/L2 correspondientes $(r=0,76, p<0,05$ en pacientes con IRA) y $(r=0,58, p<0,05$ en pacientes con IRC). No se encontró correlación entre los valores séricos de urea y creatinina y los correspondientes de amilasa y lipasa. La figura 1 muestra la relación de isoamilasas e isolipasas en los tres grupos estudiados.

\section{DISCUSIÓN}

El riesgo de pancreatitis en el curso de la enfermedad renal parece un hecho cierto. Estudios de autopsias, antes de la implantación de la hemodiálisis, demostraron evidencias histológicas de daño pancreático en pacientes urémicos (5), otros autores, también han encontrado cambios histológicos después del advenimiento de la diálisis; Vaziri y cols. describen signos de pancreatitis crónica en el $28 \%$ de los pacientes sometidos a hemodiálisis durante mucho tiempo (6); en otros estudios han encontrado evidencias histológicas de pancreatitis, tanto aguda como crónica, en el $50 \%$ de los pacientes en estadios finales de la enfermedad renal $(7,22)$.

La pancreatitis, en el curso de la enfermedad renal es una seria complicación, la mortalidad es alta (33\%), mientras que la mortalidad presentada por los pacientes no urémicos es solo del 5\%. El diagnóstico de pancreatitis en enfermos con insuficiencia renal es difícil, las enzimas pancreáticas más o menos específicas como la amilasa y lipasa, están aumentadas en el enfermo urémico, siendo la alteración de la función renal un factor importante que contribuye a su elevación sérica, incluso en ausencia de inflamación pancreática $(23,24)$. Thierry y cols. (1988) estudiaron los niveles de amilasa y lipasa en pacientes con fallo renal crónico (25); Collen et al, en 1990, además, tripsina e isoamilasas en pacientes con insuficiencia renal (10); recientemente Seno y cols. (1995) añadieron el estudio de otras enzimas como la fosfolipasa A2 y la elastasa I (12). Otros autores han realizado amplios estudios enzimáticos intentando determinar si existe una relación entre la elevación sérica de las enzimas pancreáticas y el deterioro de la función renal (26-28).

En nuestro estudio las actividades totales de amilasa y lipasa se encuentran elevadas en todos los enfermos urémicos, 

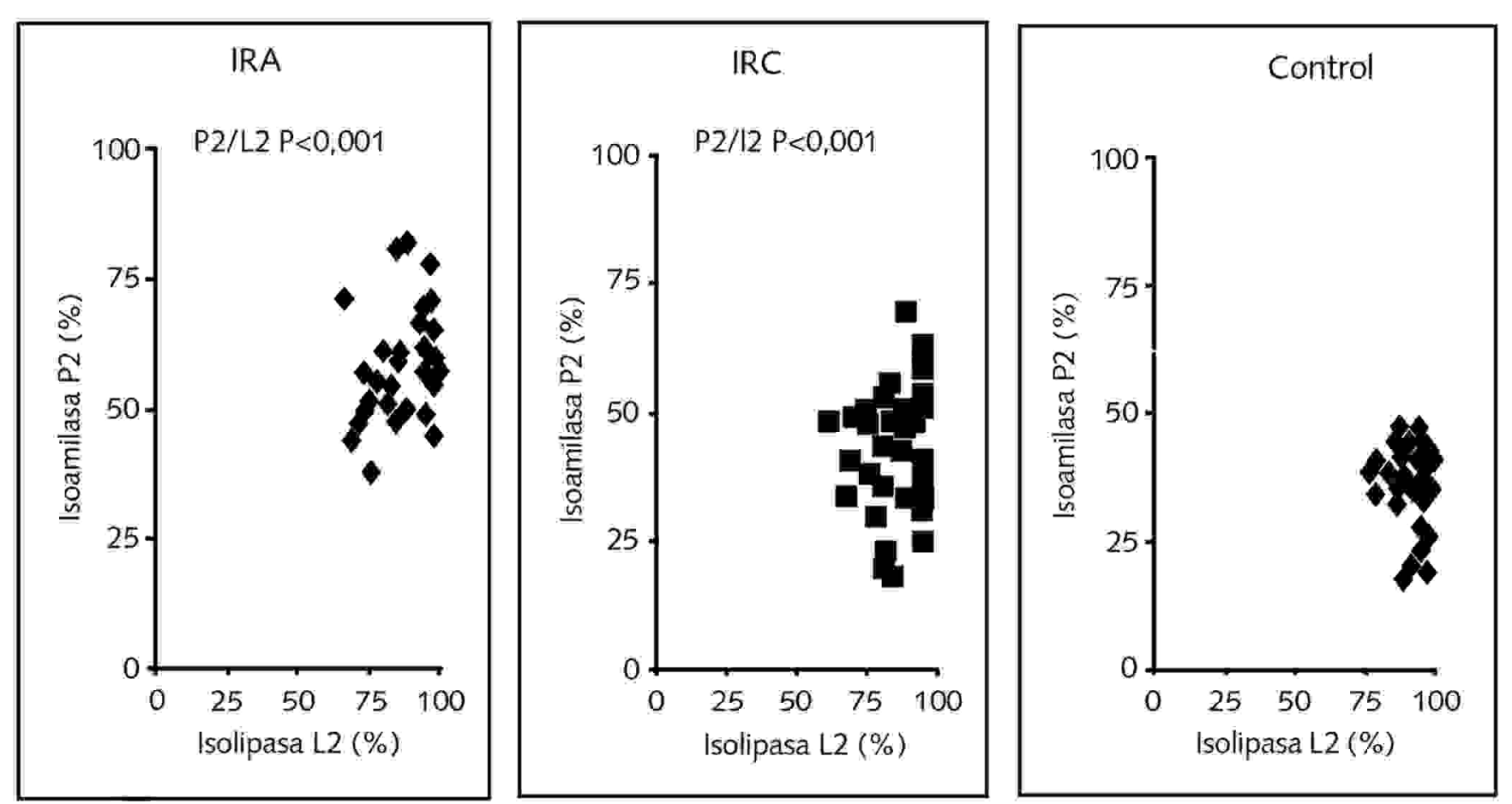

Fig. 1. Relación entre isoamilasas e isolipasas séricas en los tres grupos estudiados.

tanto agudos como crónicos, sin que ninguno de ellos presentaran signos clínicos de pancreatitis, el aumento, aunque moderado, resulta estadísticamente significativo en comparación con el grupo control.

Pero la hiperamilasemia no es especifica de la enfermedad pancreática. La amilasa sérica puede aumentar en otros procesos patológicos puesto que la enzima se encuentra en otros órganos aparte del páncreas (glándulas salivales, hígado, riñón, intestino delgado, etc.) y puede ser producida por varios tumores (carcinoma de pulmón, esófago, mama y ovario). Se sabe que las isoamilasas de tipo pancreático proceden solamente del páncreas, ya que estas isoenzimas desaparecen totalmente del suero de los pacientes pancreotomizados, por lo que, la medición de las isoamilasas de tipo pancreático son un indicador más específico de pancreatitis que la amilasa sérica total $(18,29)$.

En nuestro estudio, la hiperamilasemia encontrada en todos los pacientes con disfunción renal es debida al aumento de las isoamilasas de tipo pancreático, con un cociente $\mathrm{P} / \mathrm{S}$ superior a 0,8 . En vista de este hecho, la posibilidad de que la hiperamilasemia, en los pacientes urémicos, sea debida a una pancreatitis debe ser considerada, teniendo en cuenta además, que las concentraciones de P2 se mantienen significativamente elevadas en los pacientes crónicos, a pesar de la hemodiálisis.

La lipasa, enzima producida principalmente por el páncreas, no aumenta en respuesta a un numero tan elevado de condicionantes como la amilasa, ha sido ampliamente estudiada y utilizada como marcador bioquímico de pancreatitis aguda y su utilidad evaluada, por muchos autores, en el enfermo con insuficiencia renal (30-33). Sin embargo, se ha encontrado actividad lipolítica en otros tejidos, (hígado, intestino delgado, colon, estómago), lo que hace que la determinación de esta enzima tampoco sea enteramente específica de daño pancreático (34-37), y, por otro lado, se ha observado un aumento de la actividad sérica de lipasa en los pacientes después de la hemodiálisis, aumento que se ha atribuido a la actividad lipolítica inducida por la heparina (38).

En busca de una mayor especificidad en los resultados hemos estudiado las isolipasas L1 y L2, utilizando un nuevo método de separación electroforética, desarrollado en nuestro laboratorio, utilizando el grupo control para establecer previamente valores de referencia $(39,40)$. Del estudio de isolipasas deducimos que, la hiperlipasemia en el enfermo urémico, tanto agudo como crónico, está condicionada por el aumento de la isolipasa L1, encontrando un cociente L1/L2 superior a 0,25 .

En el estudio combinado de isoamilasas e isolipasas encontramos una correlación entre el aumento de la isoamilasa P2 y la isolipasa L1 que reflejamos, con el objeto de facilitar la interpretación de resultados, en el cociente P2/L2. El hecho de que las isoamilasas tipo P2 y las isolipasas L1 estén elevadas en el enfermo con disfunción renal podría ser importante para diferenciar a los pacientes urémicos con o sin pancreattitis.

A la vista de los resultados concluimos que tanto las isoamilasas como las isolipasas son marcadores bioquímicos eficaces en el diagnóstico de pancreatitis asociada a la disfunción renal y aunque el diagnóstico no pueda basarse exclusivamente en estos parámetros, siendo necesaria la confirmación con pruebas diagnosticas objetivas, una vez realizado éste, el estudio combinado de las isoenzimas pancreáticas y la posible variación de los perfiles isoenzimáticos en el curso de la enfermedad renal, podrían resultar de gran utilidad en relación con la frecuencia, severidad y evolución de esta grave complicación. 


\section{Bibliografía}

1. Malferther P, Staun-Olsen P, Magid E. Inmunorteactive trypsin and pancreatic isoamylase activity in serum of patients with chronic renal failure or hepatic cirrhosis. Clin Chem 1981; 27: 1655-7.

2. Rutsky EA, Robards M, Van Dyke JA. Acute pancreatitis in patients with end-stage renal disease without transplantation. Arch Intern Med 1986; 146: 1741-5.

3. Ventrucci M, Campieri C, Di Stefano M, Ubalducci GM, Li Bassi S, Di Grazia A, Giudicissi A, Festi D. Alterations of exocrine pencreas in end-stage renal disease. Dig Dis Sci. 1995; 12: 2576-81.

4. Pitchumoni CS, Arguello P, Agarwal N, Yoo J. Acute pancreatitis in chronic renal failure. Am J Gastroenterol 1996; 91: 2477-82.

5. Baggenstoss AH. The pancreas in uremia: A histopathologic study. Am J Pathol 1948; 24: 1003-18.

6. Vaziri ND, Dure-Smith B, Miller R, Mirahmadi M. Pancreatic pathology chronic dialysis patients an autopsy study of 78 cases. Nephron 1987; 46: 347-9.

7. ArakiT, Uceda M, Ogawa K, Tsuji T. Histological pancreatitis in endstage renal disease. Int J Pancreatol 1992; 12: 263-269.

8. Zachee P, Lins RL, Debroe ME. Serum Amylase and lipase values in acute renal failure. Am J Gastroenterol 1985; 31: 1237

9. Royse VL, Jensen OM, Corwin HL. Pancreatic enzymes in chronic renal failure. Arch Intern Med 1987; 147: 547-9

10. Collen MJ, Ansher AF, Chpman AB. Serum amylase in patients with renal insufficiency and renal failure. Am J Gastroenterol 1990; 85: 1377-80.

11. Buchman AL, Ament ME, Moukarzel A. Total serum Amylase but lipase correlates with measured glomerular filtration rate. J Clin Gastroenterol 1993; 16: 204-6

12. Seno T; Harada H, Ochi K, Tanaka J. Serun levels of six pancreatic enzymes as related to the degree of renal dysfunction. Am J Gastroenterol 1995; 90: 2002-5

13. Montalbo G, Carroccio A, Sparacino V. Pancreatic enzymes in chronic renal failure and transplant patients. Am J Gastroenterol 1992; 12: 211-7.

14. Junge W, Malyusz M, Ehrens HJ. The role of the kidney in the elimination of pancreatic lipase and amylase from blood. J Clin Chem Clin Biochem $1985 ; 23: 387-92$

15. Arzoglou PL, Férard G, Métais P. Differentiating two forms of plasma lipase by use of media with different ionic strengths.Clin Chem 1984; 30:360-3.

16. Panteghini M. Electrophoresis fractionation of pancreatic lipase. Clin Chem 1992; 38:1712-16.

17. Sánchez MR, Oliver C. Measurement of Pancreatic Lipase Isoforms by Agarose Electrophoretic Fractionantion. On-line Proceedings of the 5th Internet Word. Congress on Biomedical Sciences 1998 at McMaster University, Ontario, Canada (available from URL:http://www.mcmaster.ca/inabis98/cellbio/sanchez0680/index.htlm)

18. Leclerc P, Forest JC. Variations in amylase isoenzymes and lipase during acute pancreatitis, and in other disorders causing hyperamylasemia. Clin Chem 1983; 29:1020-3.

19. Lott JA, Lu CJ. Lipase isoforms and amylase isoenzymes: Assays and application in the diagnosis of acute pancreatitis. Clin Chem 1991; 37:361-368.

20. Fernandez-Conde E, Oliver C, Sanchez MR. Lipase isoforms and Amylase Isoenzymes. Combined Diagnosis Value of these Biochemical Markers in Hyperamylasemic Patients. Clin Chem Lab Med. 1999; 37:474.
21. Moreu L, Molineri LM, Fernández E. RSIGNA. Base de datos. Bioestadistica. Madrid: Horus Hardware S.A;.1990.

22. Vaziri ND, Chang D, Malekpour A, Radath S. Pancreatic enzymes in patients with end-stage renal disease maintained on hemodialysis. Am J Gastroenterol 1988; 4:410-12.

23. Berk JE, Fridhandler L, Ness RL. Amylase and isoamylase activities in renal insufficiency. Ann Intern Med 1979; 90: 351-3.

24. Levitt MD, Ellis C. Serum isoamylase measurements in pancreatitis complicating chronic renal failure. J Lab Clin Med 1993; 1: 71-7.

25. Thierry FX, Dueynes JM, Vernier I. Serum lipase and amylase levels in chronic renal failure: Interpretation of results- Effects of extra renal purification. Nephrologie 1988; 9: 263-7.

26. Hörl WH, Wanner C, Schollmeyer P, Ogawa M. Plasma levels of pancreatic secretory trypsin inhibitor in relation to amylase and lipase in patients with acute and chronic renal failure. Nephron 1988; 49: 33-38.

27. Masoero G, Bruno M, Gallo L, Colaferro S, Cosseddu D. Increased serum pancreatic enzymes in uremia: relation with treatment modality and pancreatic involvement. Pancreas 1996; 13: 350-5.

28. Lin XZ, Wang SS, Tsai YT. Serum amylase, isoamylase and lipase in the acute abdomen. J Clin Gastroenterol 1989; 11: 47-52.

29. Kolars JC, Ellis CJ, Levitt MC. Comparasion of serum amylase pancreatic isoamylase and lipase in patients with hyperamylasemia. Dig Dis Sci 1984; 29: 289-93.

30. Miller-Petersen J, Klserke M, Date F. Evaluation and comparasion of cathode trypsin-like immonoreactivity, pancreatic lipase and pancreatic isoamilase in the disgonosis of acute pancreatitis in 849 consecutive patients with acute abdominal pain. Clin Chem Acta 1986; 157: 151-65.

31. Carvana RJ, Altman R, Fowler B. Correlates of amylase and lipase in chronic dialysis patients. Int j Artific Organs 1988; 11: 454-8.

32. Nordestgaard AG, Wilson SE, Willians RA. Correlation of serum amylase levels with pancreatic pathology and pancreatitis etiology. Pancreas 1988; 3: 159-162.

33. Viel JF, Foucoult P, Bureau F, Albert A. Combined diagnostic value of biochemical markers in acute pancreatitis. Clin Chem Acta 1990; 189: 151-65.

34. Lott JA, Patel ST, Sawhney A, Kazmierczak S, Love JE. Assays of serum lipase: Analytical and clinical considerations. Clin Chem 1986; 32: 1920-32.

35. Miller IL, Lott JA. Lipase total and isoenzymes activities in normal human tissues.Clin Chem 1988; 34: 1289.

36. Apple f, Benson P, Preese L. Lipase and prancreatic amylase activities in tissues and in patients with hyperamylasemia. Am J Clin Pathol 1991; 96: 610-4

37. Lessinger JM, Arzoglou PL, Ferard G. Evidence for multiple forms of pancreatic lipase in human plasma. Adv Clin Enzymol 1986; 3: 139-50.

38. Montalto G, Soresi M, Carroccio A, Galione A, Lorello D. Influence of haemodialysis on lipase activity. Eur J Clin Chem Clin Biochem 1997; 35: 237-8.

39. IVB Nomenclature Committee. Enzyme nomenclature, New York: Academic Press, Inc. 1984

40. Sánchez MR, Oliver C. Nuevo método para el fraccionamiento electroforético de lipasa pancreática. Relación con los perfiles isoenzimáticos de amilasa en pacientes hiperamilasemicos. Rev Diagn Biol 1997; 46: 225-231. 